\title{
Improvements to Bison Validation Base for LWR Fuel
}

\section{L3:FMC.FUEL.P19.09}

Stephanie A. Pitts, INL Russell J. Gardner, INL Al Casagranda, INL Benjamin W. Spencer, INL Daniel J. VanWasshenova, INL Dylan J. McDowell, INL Giuseppe Rota, INL Richard L. Williamson, INL

August 30, 2019

REVISION LOG

\begin{tabular}{|c|c|c|l|}
\hline Revision & Date & Affected Pages & \multicolumn{1}{c|}{ Revision Description } \\
\hline 0 & $08 / 30 / 2019$ & All & Initial Release \\
\hline & & & \\
\hline & & & \\
\hline & & & \\
\hline
\end{tabular}

\section{Document pages that are:}

Export Controlled NONE

IP/Proprietary/NDA Controlled NONE

Sensitive Controlled NONE

This report was prepared as an account of work sponsored by an agency of the United States Government. Neither the United States Government nor any agency thereof, nor any of their employees, makes any warranty, express or implied, or assumes any legal liability or responsibility for the accuracy, completeness, or usefulness of any information, apparatus, product, or process disclosed, or represents that its use would not infringe privately owned rights. Reference herein to any specific commercial product, process, or service by trade name, trademark, manufacturer, or otherwise, does not necessarily constitute or imply its endorsement, recommendation, or favoring by the United States Government or any agency thereof. The views and opinions of authors expressed herein do not necessarily state or reflect those of the United States Government or any agency thereof.

\section{Requested Distribution:}

To: FMC FA Lead

Copy: CASL PM 


\section{Improvements to Bison Validation Base for LWR Fuel}

Stephanie A. Pitts, Russell J. Gardner, Al Casagranda, Benjamin W. Spencer, Daniel J. VanWasshenova, Dylan J. McDowell, Giuseppe Rota, and Richard L. Williamson

Computational Mechanics and Materials Department

Idaho National Laboratory

P.O. Box 1625

Idaho Falls, ID 83415-3840

August 30, 2019 


\section{Contents}

1 Introduction 2

2 Updates to Existing Bison LWR Validation Cases 3

2.1 Standardization of Common LWR Output Quantities . . . . . . . . . . . . . . . . . . . . 3

2.2 Migration of Validation Cases to Tensor Mechanics . . . . . . . . . . . . . . . . . . . . . 4

2.2.1 Input File Migration Process . . . . . . . . . . . . . . . . . . . . . . . 5

2.2.2 Standardized Migration Simulation Results Script . . . . . . . . . . . . . . . . . . . . . 7

2.3 Code Reliability Improvements . . . . . . . . . . . . . . . . . . . . . . . . . . . 7

2.3.1 Resolution of Hard-Coded Options in Solid Mechanics . . . . . . . . . . . . . . . . . . 7

2.3.2 Ensured Compatibility between Mechanics Modules . . . . . . . . . . . . . . . . . . 8

2.3.3 Influence of Timestep on Zircaloy Primary Creep . . . . . . . . . . . . . . . . . . . . . 8

2.4 Investigation of Additional Physics Models . . . . . . . . . . . . . . . . . . . . . . . 10

2.4.1 New Physics Models Considered . . . . . . . . . . . . . . . . . . . . . . . . . . 10

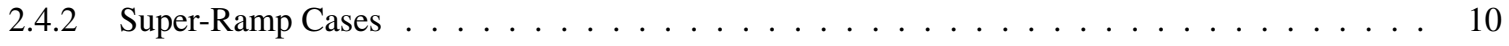

2.4 .3 Risø GE7 Case . . . . . . . . . . . . . . . . . . . . . . . . 11

3 Revisions to LWR Validation Case Documentation 14

3.1 Revise LWR Validation Documentation . . . . . . . . . . . . . . . . . . . . . . . . . . 14

3.2 Standardization of Assessment Case Plots . . . . . . . . . . . . . . . . . . . . . . 15

3.2.1 Matplotlib Style-File . . . . . . . . . . . . . . . . . . . . . . . . . 15

3.3 Modernize Plots in Assessment Cases Documentation . . . . . . . . . . . . . . . . . . 16

3.3.1 Initial Automated Plot Generation Development . . . . . . . . . . . . . . . . . . . . 17

4 Development of New LWR Validation Cases 18

4.1 IFA-629.4 High Burnup Fuel Case . . . . . . . . . . . . . . . . . . . . . . . . . . . 18

4.1 .1 Experimental Description . . . . . . . . . . . . . . . . . . . 18

4.1.2 Bison Computational Model . . . . . . . . . . . . . . . . . . . . . . 18

4.1.3 Results and Comparisons . . . . . . . . . . . . . . . . . . . . . . 20

5 Expansion of User Support Documentation $\mathbf{2 4}$

5.1 Revised Bison Getting Started Page . . . . . . . . . . . . . . . . . . . . . . . . . . 24

5.2 Tensor Mechanics Migration Guide Development . . . . . . . . . . . . . . . . . . . . . . 25

6 Summary and Future Work 27

7 Acknowledgments 27 


\section{Introduction}

The Bison fuel performance code is being developed by Idaho National Laboratory (INL) to analyze a wide range of fuel forms and operating conditions with the flexibility to conduct simulations in 1D through 3D geometries. Bison plays an important role in the development of advanced fuel with improved accident tolerance for existing light water reactors (LWRs), the improved understanding of mechanisms in fuel designs in a wider range of operating conditions, and the facilitation of future development of fuel for advanced reactor designs. Additionally, industry interest in advanced fuels has also driven Bison development for advanced fuels in the last few years. All of these factors combine to create a potential increase of new Bison users. These new users will be interested in the Bison LWR validation base for examples of Bison's capabilities and how to use Bison. Current users also rely on the Bison validation base as a demonstration of the best practices settings and material behavioral models.

Bison, both through direct updates to the Bison source code and to the nearly daily changes and improvements in the underlying MOOSE framework source code, undergoes nearly continuous changes. It is necessary that these source code changes are reflected with updated input files in the Bison validation base. Periodic updates of the validation case input files should also be accompanied by a review and revision of the validation case documentation on which our users rely. Therefore our efforts under this milestone were guided by two objectives:

1) Update the Bison LWR validation input files to use current Bison simulation settings, and

2) Enhance the ease-of-use for Bison through improved documentation

These objectives build on the goals identified FY18 CASL milestone reports. During FY18 we addressed issues with the robustness of Bison and identified a need to migrate the assessment suite to the more reliable tensor mechanics module system and set a goal of standardizing the Bison input files [1]. The FY18 CASL milestone report on updates to the Bison documentation system [2] listed continued development of user-focused guides and examples along with revisions to the validation case documentation as future development goals.

During FY19 we have separated these two objectives into four different focus areas for our work on this milestone. Our first two focus areas target the existing Bison validation suite: update the existing LWR validation cases and the accompanying documentation. The second two focus areas center on adding new capabilities: a new Bison LWR validation case and expansion of user support documentation. In the following report each of these four focus areas are discussed as a separate section. We conclude the report with a summary of potential future work to build on the efforts of this year. 


\section{Updates to Existing Bison LWR Validation Cases}

The input file improvements to the existing Bison LWR assessment suite represent the majority of our efforts within this milestone. Given the role played by these validation case input files as examples for new and current users, ensuring that these cases represent the current best practices for Bison simulations is key. Our efforts included standardization of the input files, migration of the input files to the more robust and reliable tensor mechanics module system and corresponding reliability improvements to the older solid mechanics module system, and an investigation into using additional physics in the simulations.

\subsection{Standardization of Common LWR Output Quantities}

As identified in the FY18 CASL milestone on improving robustness in Bison simulations [1], standardization of the Bison input files was one of our priorities for this milestone. Given the consistency in LWR output quantities, we focused on standardizing the outputs of a Bison simulation.

The standardization involved both the method of collecting the simulation results, including the location on the simulation mesh, and the name given to the output simulation data. A total of ten scalar postprocessors and two vector postprocessors were identified as appropriate for all LWR fuel simulations. The standard quantities collected from the fuel mesh block are

- average_centerline_fuel_temperature

- fission_gas_generated

- fission_gas_released

- fission_gas_released_percentage

- pellet_volume

- maximum_fuel_elongation

- fuel_outer_radial_displacement_FINAL

and those associated with the cladding mesh block are

- average_interior_clad_temperature

- plenum_temperature

- plenum_volume

- maximum_clad_elongation

- clad_radial_displacement_FINAL

The notation FINAL at the end of the clad and fuel radial displacement quantities indicates that these data are collected only at the end of the simulation. These two vector postprocessor outputs collect the value of the radial displacements along the axial length of the fuel rod.

The natural path for implementing these output standards was the creation of an action. This action, termed the StandardLWRFuelRodOutputs action, automatically creates all twelve of the outputs listed above. This action also increases the usability of Bison by replacing over 60 lines in the input file with just 6 lines.

Given that not every Bison LWR validation case involves both the fuel and the cladding mesh blocks, an option to select the fuel rod component of interest is given in the StandardLWRFuelRodOutputs action. When this option is set to either fuel or clad, the action will only create those outputs associated with that component of the fuel rod. An example of the different action usage options is shown in Figure 1.

The standard output quantities and names created by the StandardLWRFuelRodOutputs action are instrumental in creating automated plots to compare Bison simulations, as discussed in Section 2.2.2. Both the plotting script and the StandardLWRFuelRodOutputs action are utilized in the process of migrating Bison validation cases from the older solid mechanics module system to the new, more reliable, tensor mechanics module system. 


\title{
Example Input File Syntax
}

\begin{abstract}
The default behavior of the StandardLWRFuelRodOutputs action will create a standard set of outputs
\end{abstract} for both the fuel mesh block component of the rod and the cladding:

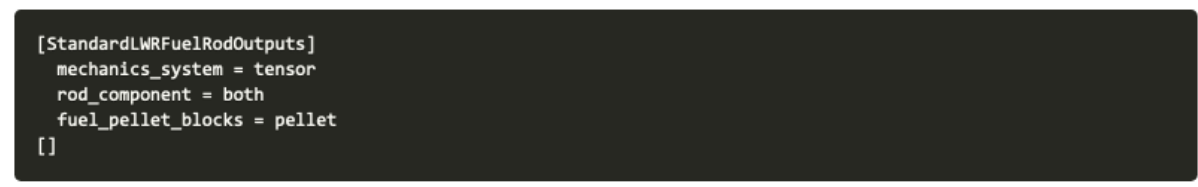

(test/tests/standard_lwr_outputs_action/mini_complete_rod.i)

It is also possible to use the StandardLWRFuelRodOutputs action to generate outputs for only the fuel or only the cladding, depending on the rod_component selected. Here an example of the StandardLWRFuelRodOutputs action for a fuel pellet only simulation is shown:

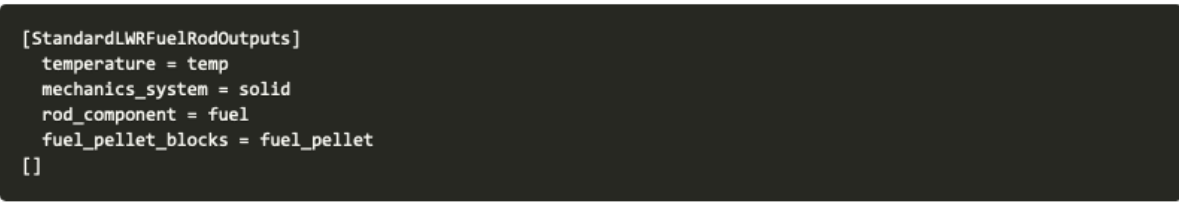

(test/tests/standard_Iwr_outputs_action/pellet_only.i)

Figure 1: An excerpt from the Bison documentation manual, this figure demonstrates the methods available to create outputs associated with either both fuel rod components or only a single component.

\subsection{Migration of Validation Cases to Tensor Mechanics}

The change to use the tensor mechanics module as a replacement for the older solid mechanics module as the basis for all material mechanics code provides several advantages. The newer tensor mechanics module offers more flexibility to include anisotropy, better correspondence to mathematical notation for formulas, and more integration with other MOOSE physics modules.

Critically, the tensor mechanics module also enables better control over which mechanics models are included in the simulation. This control is a direct consequence of the plug-n-play structure of tensor mechanics, in which each mechanics physics component is separated into individual models. This capability ensures that only the physics which a user selects in the input file are implemented and prevents mechanics material blocks from overriding other mechanics material block settings in the input file.

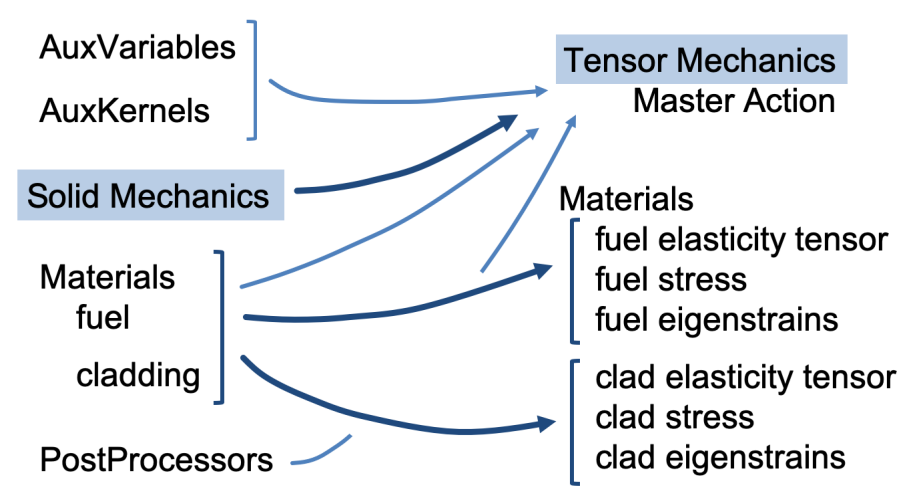

Figure 2: The tensor mechanics migration efforts focus on replicating the material behavior model functionality provided by the solid mechanics kernels and materials in the tensor mechanics module. Those auxvariables, auxkernels, and postprocessors which involve tensor quantities, such as stress and strain, must also be changed. 


\subsubsection{Input File Migration Process}

Our efforts in migrating the assessment case input files center on replicating the functionality provided by the solid mechanics kernel and materials with the associated tensor mechanics models. The migration process, an overview of which is shown in Figure 2, also involves changing the auxvariables, auxkernels, and postprocessors which are used to output stress and strain data.

Creating a tensor mechanics input file is a process of carefully following the provided guidelines, described in Section 5.2. The mechanics migration of a Bison LWR assessment case is only considered successful when the plots of eight different physical metric quantities align. These quantities are

- Average fuel centerline, plenum, and interior cladding temperatures,

- Fuel pellet and plenum volumes,

- Fission gas released percentage, and

- Fuel and cladding exterior radial displacements at the end of the simulation.

These quantities were selected because they represent the different field variables, temperature and displacement, which appear in the governing equations for a Bison simulation. Additionally these quantities represent the measurements which can appear in the experimental data for LWR validation cases. An example of the successful migration com-

Figure 3: The migration of a Bison validation case from the solid mechanics system to the tensor mechanics system is considered successful when plots of the simulation results align. Here a subset of the comparison plots for the Ris $\emptyset$ AN3 validation case are shown to demonstrate the alignment of the solid mechanics quantities, indicated with red dots, with the corresponding tensor mechanics quantities, given as black squares. 
parison of some of these quantities is shown in Figure 3. Note that the larger number of red dots at right side of the plots in Figure 3 indicates only that the solid mechanics simulation took more time steps to converge at the end of the simulation. The final solution of the solid mechanics and tensor mechanics simulation is the same, as shown by the aligned markers for the final timestep.

However, the results of the new tensor mechanics input file simulation may not match the previous solid mechanics graphical outputs for a number of reasons. The disagreements do not directly imply an incorrect solution of either model, but instead highlight the changes in the calculations of quantities and methods used. Debugging the contrasting methods is a time-consuming process but is necessary to determine where the disparities occur.

As a first step, checking to make sure all inputs to the simulations are identical helps to eliminate any error in the input files. This can be determined by using:

$$
\text { projects/bison/bison-opt - -show-input -i input_file.i > temp.out }
$$

Creating a text file of all inputs, given and hidden, can then be used to compare the solid mechanics and tensor mechanics versions for the simulations. Checking for dissimilarities and changing one component at a time is the slow but scientific process of making the models agree as much as possible before moving on. Using an editor such as Atom (https://atom.io), with the "Split Diff" setting, can help speed up this comparison process. Disagreement in parameters such as cold_work_factor, which are assumed to follow the defaults of the models, have been found in this manner.

Additional differences are highlighted by comparing specific parameter values as a function of time. Using the solid mechanics and tensor mechanics CSV files, spreadsheets can be created for the respective cases. Creating plots for each case, as a function of time, will show where the divergence between models occur initially. This leadoff time can be compared across plots to see which variable could be the nonconforming factor. Once a possible deviance is discovered, parameters can be isolated in the input files, to further distinguish the results. Logically progressing through permutations of the parameter's on/off states and comparing plots determines the cause of variance. Once a prime candidate is identified, we can develop a specific regression test to further determine the cause(s). This method has highlighted a discrepancy in primary creep for the MechZry model. Further investigation within the code will follow once the problem is better understood.

Given these challenges, the migration of the Bison LWR assessment suite is ongoing, and efforts to migrate the entire Bison assessment suite to the tensor mechanics system will continue in the next FY. The current tensor mechanics migration status of the Bison LWR assessment suite shown in Figure 4, with 64\% of the assessment suite implemented in a tensor mechanics input file. As of this writing only $36 \%$ of the Bison assessment suite has not yet been migrated to the tensor mechanics system.

Figure 4: Current status of the migration of Bison LWR validation and benchmark cases to tensor mechanics. The middle category, mechanics pairs, indicates validation cases which exist as both a solid mechanics input file and a second tensor mechanics input file. 
Pairs of the solid mechanics input file and the corresponding tensor mechanics input file are retained for several validation cases as examples to guide future migration. These different input file pairs demonstrate the correct migration for the several different simulation options implemented throughout the Bison assessment suite.

\subsubsection{Standardized Migration Simulation Results Script}

Given the importance of plotting simulation results in the migration process, we have developed a plotting script to compare the results of the solid mechanics and tensor mechanics simulations using matplotlib and pandas. This plotting capability speeds up the process of verifying the input file migration and ensures that the result comparison plots are generated in a standard and consistent manner. The script, SMvTM_plots.py, is stored in the projects/bison/scripts directory.

Leveraging the StandardLWRFuelRodOutputs action, which creates output quantities with consistent names, we developed this plotting script to select and plot the eight comparison quantities listed in Section 2.2.1. This plotting script includes the flexibility to compare the simulation results stored in either the same or different directories, and, like the StandardLWRFuelRodOutputs action, to generate comparison plots for either the complete fuel rod, the fuel only, or only the cladding. Guidelines for using the plotting script are included within the migration documentation, Section 5.2. Examples of the plots generated with this script are shown in Figure 3.

In addition to being utilized during the input file migration process, this script is also employed to verify the mechanics input file pairs in the Bison LWR assessment suite. Validation cases which exist as solid mechanics and tensor mechanics pairs have an additional file included in the repository, called post. This post file can be used to call this plotting script at the conclusion of the nightly Bison assessment suite runs to generate the current version of the comparison plots.

\subsection{Code Reliability Improvements}

An important consequence of the assessment case input file migration is the identification of code discrepancies between tensor mechanics and solid mechanics. The comparison of the integral Bison fuel rod simulations for the two different mechanics modules provides a more thorough review of the Bison source code than is sometimes possible with the short regression tests. Often these discrepancies were found within the solid mechanics module while the tensor mechanics module, due to the plug-n-play design, was not subject to the same reliability issues which affected the solid mechanics module. The steps taken to resolve these code discrepancies and to ensure matching results between the solid mechanics module and tensor mechanics module input files are discussed below.

\subsubsection{Resolution of Hard-Coded Options in Solid Mechanics}

Because of the centralized nature of the solid mechanics module materials, many options for multiple different behavior models must be set within the single input file block. Throughout the tensor mechanics migration process we discovered insufficient error checking in the solid mechanics materials which allowed for incompatible parameters to be set simultaneous in the input file. In these instances the solid mechanics code would silently select a single model to use and override the user settings. We have modified the solid mechanics code to improve the input file error checking.

Similarly we discovered that some of the solid mechanics inelastic material models were not properly integrated with updates to the elasticity tensor. These inelastic material models were using a static value from the initial elasticity tensor. This bug in the solid mechanics module class was causing incorrect results. We have resolved this issue by modifying the solid mechanics module class to retrieve the update elasticity tensor values. 


\subsubsection{Ensured Compatibility between Mechanics Modules}

The method for calculating the contribution of strain due to volumetric eigenstrains differs between the tensor mechanics and solid mechanics systems. During FY19 we identified the source of this disparity: a difference in the model calculation of the volumetric eigenstrains in the two mechanics systems. The solid mechanics module treats the volumetric eigenstrain in a manner consistent with a small linearized strain formulation

$$
\epsilon^{s m}=\left(\epsilon_{v}+1\right)^{1 / 3}-1
$$

while the tensor mechanics module accounts for finite strain by including a logarithmic term in the volumetric eigenstrain calculation

$$
\epsilon^{t m}=\log \left[\left(\epsilon_{v}+1\right)^{1 / 3}\right] .
$$

The logarithmic expression used by the tensor mechanics module is more widely applicable to different simulations, including large nonlinear deformation simulations.

To ensure matching simulation results between solid mechanics and tensor mechanics input files, we have added a tensor_mechanics_compatibility option to the solid mechanics volumetric eigenstrain models. The affected solid mechanics models include relocation and relocation recovery in $\mathrm{UO}_{2}$ and volumetric swelling in $\mathrm{UO}_{2}$. This compatibility option forces the solid mechanics models to apply Equation 2 when calculating the volumetric eigenstrains.

\subsubsection{Influence of Timestep on Zircaloy Primary Creep}

We uncovered a potential issue with the calculation of the primary creep during the migration of the HBEP BK370 assessment case. The comparison of the radial cladding strains at the end of the solid mechanics and tensor mechanics simulations shows a difference which we traced to the Zry primary creep model in conjuction with irradiation creep, as shown in Figure 5.

(a) Zry primary creep model activated.

(b) Zry primary creep model inactive.

Figure 5: Cladding radial displacement comparisons between the solid mechanics (SM) and tensor mechanics (TM) simulations for HBEP with and without Zry primary creep active in Bison simulation.

The time history of the cladding displacement is also helpful in diagnosing the issue and is shown in Figure 6. As one can see, the solid mechanics and tensor mechanics solutions match throughout the simulation when Zry primary creep is inactive; however, if the Zry primary creep model is active, the solid mechanics and tensor mechanics simulation results begin to differ later in the analysis. Focusing on the early stage of the simulation shows that the time stepping of the solid mechanics and tensor mechanics analyses are different for both with and without the Zry primary creep model active. At some later point in the analysis the differences grow and become obvious. 

(a) Zry primary creep model activated.
(b) Zry primary creep model inactive.

Figure 6: Cladding radial displacement comparisons between he solid mechanics (SM) and tensor mechanics (TM) as a function of time for HBEP with and without Zry primary creep active in Bison simulation.
(a) Zry primary creep model activated.
(b) Zry primary creep model inactive.

Figure 7: Cladding radial displacement comparisons between the solid mechanics (SM) and tensor mechanics (TM) in the initial stage of the HBEP simulation with and without the Zry primary creep model active. Note the substantial increase in the number of time steps in the solid mechanics simulation when the Zry primary creep model is inactivated.

The source of this deviation in the solutions is still being investigated. The HBEP BK370 assessment case is too large to use in this investigation process. Instead we are currently modifying the solid mechanics and tensor mechanics zircaloy creep regression tests so that these regression tests exhibit similar behavior to the assessment case in Figures 6 and 7. These modifications will allow us to use the regression tests in the investigation of the zircaloy primary creep model differences between the solid mechanics and tensor mechanics module systems. 


\subsection{Investigation of Additional Physics Models}

Motivated by the gap between experimental measurement data and Bison simulation results for PCMI cases, as discussed within [3], we examined the impact of including additional physics models for the fuel behavior in a small set of Bison assessment cases. We have selected LWR validation cases with a power ramp after a base irradiation to investigate these physics models because the loading history, with the power ramp, is numerically more challenging and because we anticipate a larger impact on the final simulation results from these physics models.

\subsubsection{New Physics Models Considered}

We focused on adding inelastic behavior models to the fuel, which was previously model with only elastic behavior in the Bison simulations. These new physics models, listed below, can more accurately capture the deformation behavior of the fuel pellets and thus may improve the Bison simulation results when compared to the experimental data measurements. Since the cladding is already modeled with inelastic materials, the addition of these fuel behavior models ensures that the entire fuel rod is modeled with inelastic behavior models. All of these models listed below are implemented within the tensor mechanics module system.

- UO2CreepUpdate - Calculates the secondary thermal and irradiation creep for $\mathrm{UO}_{2} \mathrm{LWR}$ fuel.

- ComputeSmearedCrackingStress - Computes the fuel stress using a fixed smeared cracking model and will relax the material based on the softening model used once a threshold stress is reached.

- UO2RelocationEigenstrain - This model accounts for cracking and relocation of fuel pellet fragments in the radial direction and is necessary for accurate modeling of LWR fuel. This model also accounts for fuel relocation recovery.

The theory and implementation of these models is discussed in the Bison documentation [4] along with usage examples from the regression test input files.

\subsubsection{Super-Ramp Cases}

The Studsvik Super-Ramp project [5] investigated the behavior of typical LWR test fuel rods when subjected to power ramps, after base irradiations conducted to medium burnups. The project consists of 28 fuel rods for the PWR subprogram and 16 for the BWR subprogram. For our purposes here only the PWR subprogram was considered.

The PWR test fuel rods ( $\mathrm{UO}_{2}$ pellets with Ziracaloy-4 claddings) were tested using high ramp rates. The main goal of the PWR Super-Ramp subprogram was to establish the damage threshold for the standard design PWR rods, with ramp tests performed at burnups above $30 \mathrm{MWd} / \mathrm{kgU}$.

The irradiation experiments have been performed over 6 groups of PWR fuel rods, with different design specifications and different characteristics of both the fuel and the cladding materials. The rods were base irradiated in the KWO (Obrigheim, Germany) and BR-3 (Mol, Belgium) commercial reactors, with linear heat rates between 9 and $27 \mathrm{~kW} \mathrm{~m}^{-1}$ and burnups between 28 and $45 \mathrm{MWd} / \mathrm{kgU}$. Subsequently, the test rods underwent ramp tests in the R2 experimental reactor (Studsvik, Sweden).

These LWR validation case input files were originally committed to the Bison assessment suite repository with a specific interest in the fission gas release results. That being the case the fuel was modeled as elastic and with a manually controlled relocation model. Since these cases were originally committed, there have been many improvements to the fuel mechanical models available in Bison. While migrating the inputs to the tensor mechanics system we elected to complete a full revisit of these cases and add new, available Bison physics.

The Super-Ramp experiments examined more than just fission gas release. Mechanical examinations such as end of life profilometry and rod elongation were also preformed on some of these rods. The additional physics models, Section 2.4.1, will allow for some addition assessment comparisons against these profilometry and rod elongation experimental data. 


\subsubsection{Risø GE7 Case}

The Ris $\varnothing$ GE7 experiment entails a base irradiation of the fuel segment ZX115 in the Quad Cities-1 BWR over four reactor cycles and a later bump irradiation in the test reactor DR3 at Risø, under BWR conditions. The bump test was run to a pin average linear power of $355 \mathrm{~W} / \mathrm{cm}$ and a final average burnup of $4.17 \%$ FIMA.

We have investigated adding the additional physics models, listed in Section 2.4.1 to the Bison Ris $\emptyset$ GE7 simulation. As with the Super-Ramp simulations, our aim was to improve the Bison simulation predictions of the final cladding displacements and better match the experimental data. We conducted this investigation in two stages: in the first stage we examined the impact of individual models, and in the second stage we examined the effect of coupling various individual physics models. We evaluated the Bison simulation results from both of these stages against the experimental measurements of the cladding displacement and of the fission gas released percentage.

In the first stage of our investigation we ran individual simulations with each new fuel physics model alone to examine the separate impact of each model on the Bison results. These results are shown in Figures 8 and 9. In both of these figures we compare the results from these separate models against the original elastic fuel simulation results and the experimental measurement data.

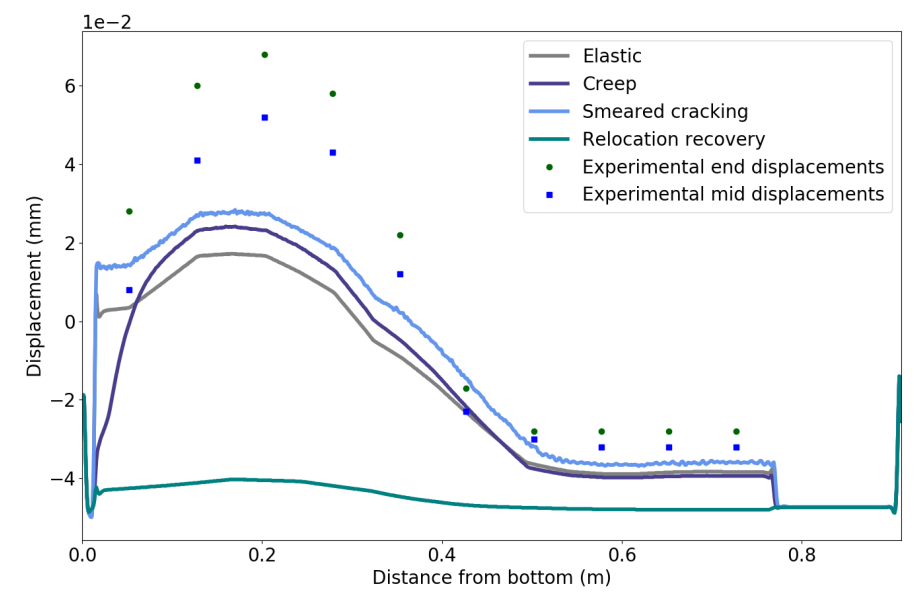

Figure 8: The variance of outer cladding radial displacement after power bump as predicted with separated physics simulations demonstrates that both the creep and smeared cracking models increase the cladding displacement while the relocation recovery model significantly reduces the cladding displacement values.

Figure 8 shows the outer cladding displacements in the radial direction as a function of axial position along the fuel rod. From the simulation with the UO2CreepUpdate model we observe a smoothing effect on the cladding displacement at the lower end of the fuel rod. This smoothing trend is better aligned with the parabolic nature of the experimental cladding displacement curves. The ComputeSmearedCrackingStress model simulation produces cladding displacement predictions which are the closest to the values of experimental data. The dramatic effect of the UO2RelocationEigenstrain model on the cladding diameter is immediately noticeable. This relocation recovery model, which recovers $50 \%$ of the relocation strain experienced by the fuel mesh block, significantly reduces the final fuel displacement. As a result the cladding experiences a significant creep-down when compared to the other fuel physics models and the experimental data. Nonetheless all three physics models demonstrate cladding displacement predictions below the experimental data, motivating the study of coupled fuel models.

We also examine the impact of these different fuel behavior models on the fission gas release predictions, Figure 9. Note that in this figure the fission gas release percentage is shown only during the power ramp period such that the starting time is set to correspond to the beginning of the bump test. The quantity of interest here is the fission gas released percentage: the quantity of released gas over the total fission gas production in the fuel. In the experiment this value, evaluated by puncturing the fuel rod, was measured as $14.4 \%$.

While the UO2CreepUpdate model does not demonstrate any significant impact on the fission gas released percentage 
when compared with the elastic fuel model, the ComputeSmearedCrackingStress model leads to a lower fission gas discharge. This lower released gas value is a consequence of the reduced gap between the fuel and cladding, as evidenced by the larger clad displacements driven by this fuel model, Figure 8 . The reduced gap between the fuel and cladding results in better thermal transfer of heat from the fuel. Since the fission gas release is primarily a temperature-driven phenomena, the reduced gap leads to a reduced fission gas release. Conversely, the relocation recovery model within UO2RelocationEigenstrain causes the fuel to move away from the cladding and thus increases the gap between the cladding and fuel. This wider gap results in lower thermal conductivity, driving up the temperature in the fuel. The higher fuel temperature caused by the relocation recovery model causes a higher fission gas release prediction.

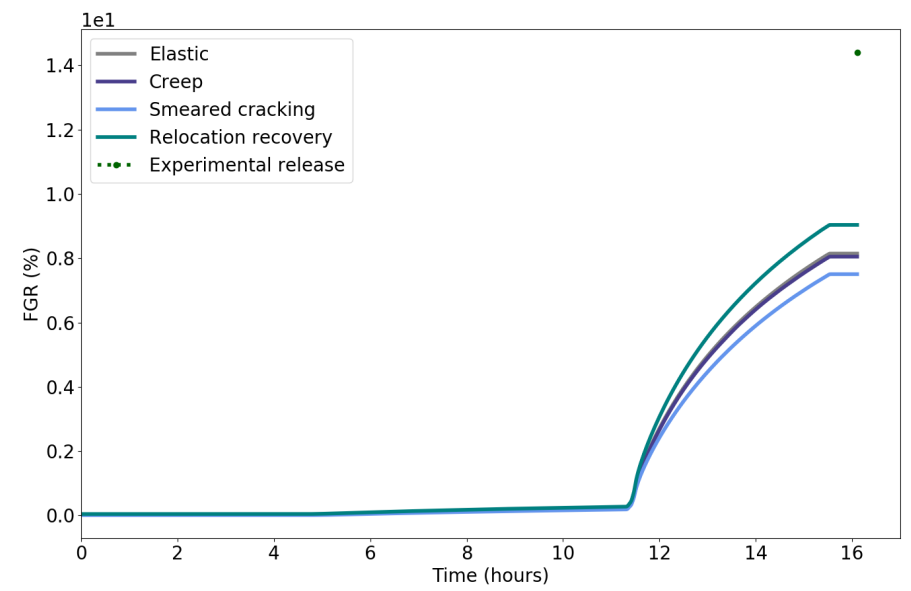

Figure 9: Fission gas released percentage after power bump as calculated with separate physics models shows that the relocation recovery model increases the fission gas released while the smeared cracking model reduces the amount of fission gas released.

In the second stage our our investigation we examined the impact of two different model couplings: the first set consists of the ComputeSmearedCrackingStress and UO2CreepUpdate models, and the second set consists of all three models listed in Section 2.4.1. We again examine the impact of these coupled models on the cladding displacement predictions, Figure 10, and on the released fission gas calculations, Figure 11.

In Figure 10 we observe that the displacement predictions from the coupled ComputeSmearedCrackingStress and UO2CreepUpdate models follow the trend of the individual smeared cracking model; however, we note a smoothing of the predicted displacement curve near the lower end of the fuel rod. We also note the presence of oscillations in the displacement predictions, which warrant further investigation. The cladding displacements predicted by the coupling of all three models are significantly affected by the fuel recovery that enables a stronger cladding creep down; nevertheless, the smeared cracking contribution does raise the displacement prediction slightly.

With respect to the fission gas release predictions, similar trends are observed with the coupled models, Figure 11, as with the individual models, Figure 9. In the case of the combined ComputeSmearedCrackingStress and UO2CreepUpdate models the prediction of the fission gas release mirrors that of the smeared cracking model alone. We surmise that in the simulation involving all three additional physics models the impact of the smeared cracking and relocation recovery models balance each other out, creating a negligible change from the prediction of fission gas release by the original elastic model. 


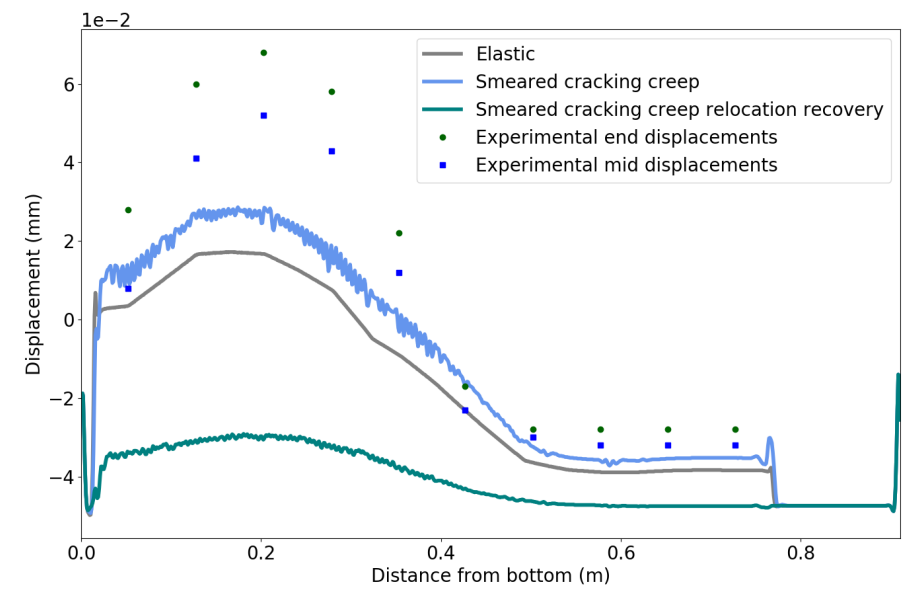

Figure 10: The outer cladding radial displacement predictions after power bump as calculated with two sets of coupled physics models indicate that the creep model softens the displacement curve near the lower end of the fuel rod while the smeared cracking and relocation recovery models dominate the overall response.

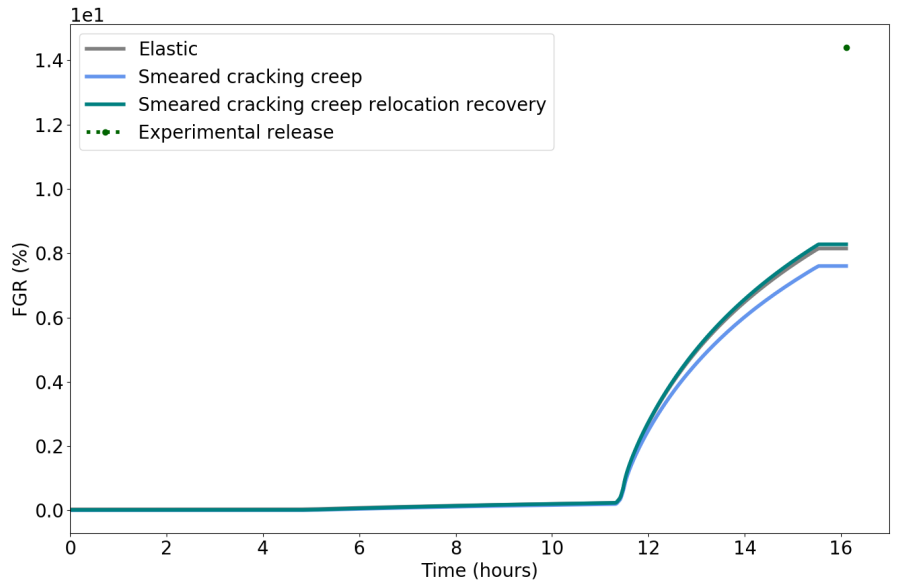

Figure 11: Fission gas released percentage after power bump as calculated with two sets of coupled models demonstrates a balance between the smeared cracking and relocation recovery model impact on the simulation results.

In general the addition of these new physics models to the Bison simulation of the Ris $\varnothing$ GE7 fuel rod results in improvements with matching the experimental cladding displacements after the bump test. The ComputeSmearedCrackingStress and UO2CreepUpdate models produced cladding displacement predictions most aligned with the experimental data. Nevertheless, future efforts should involve further analysis of the relocation recovery capability. The relocation recovery represents a critical component of the fuel behavior, yet, at present, the use of the relocation recovery model worsens the cladding displacement predictions. Once these issues are resolved, these models can be applied to other PCMI fuel rod simulations in the Bison LWR assessment suite. 


\section{Revisions to LWR Validation Case Documentation}

A necessary accompaniment to the Bison LWR assessment suite updates, Section 2, is the improvement of the associated documentation. Current validation case documentation is required to ensure users can understand the updated input files: the Bison documentation is the central resource for all users. Our efforts on this milestone leveraged the transfer of the Bison LWR assessment suite documentation from LaTeX format to the online Bison documentation system [6]. The Bison online documentation system is built on MOOSEDocs [7]. This online system performs automatic updates to the documentation as the Bison and MOOSE source code changes. Nonetheless, additional effort is required to ensure the more static Bison assessment case documentation remains current with the Bison source code.

In this section we discuss the revisions to the documentation of the existing Bison LWR assessment suite, including updates to reflect the use of tensor mechanics material models, standardization of the simulation result plots, and modernization of the simulation result plots.

\subsection{Revise LWR Validation Documentation}

With the goal of ensuring that the documentation matches the input files migrated to tensor mechanics, our first revisions to the Bison LWR assessment case documentation focused on updating the behavioral models list for each assessment case. For those assessment cases with input files that have been converted to tensor mechanics, we have updated the associated documentation to reflect the tensor mechanics modules used in the simulation. At the same time we have taken steps to ensure all the material models for both the fuel and the cladding are listed in the instances where the older documentation gave only the behavioral models applied to the fuel. A comparison between the older documentation and the revised documentation for two similar Ris $\varnothing$ assessment cases is shown in Figure 12.

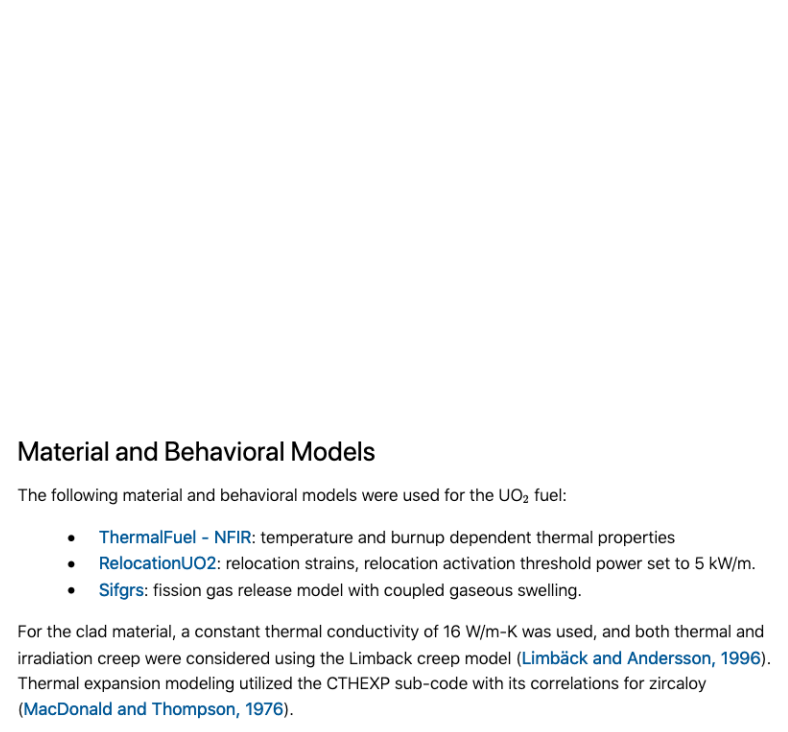

(a) Outdated list of solid mechanics materials, Ris $\varnothing$ AN2 case

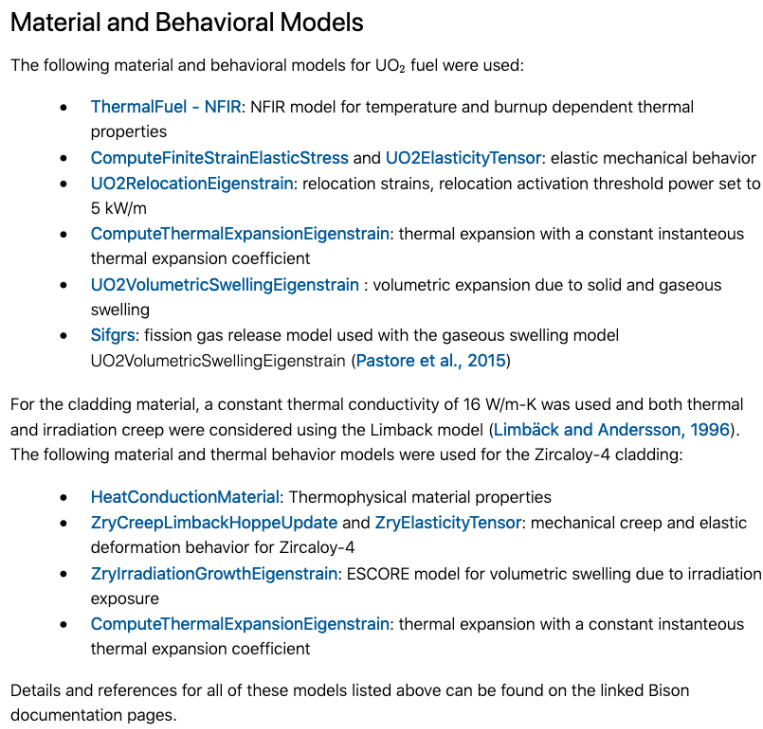

(b) Updated list of the tensor mechanics models, Ris $\varnothing$ AN3 case

Figure 12: Revisions to the Bison LWR assessment case documentation have included updating the list of material models to reflect the complete set of tensor mechanics cases used in the migrated input files.

As part of updating the LWR validation case documentation we also conducted routine maintenance throughout FY19 to ensure the correct rendering of the online documentation system. These maintenance tasks included updating the formatting of equations, citations, and figure placement as the underlying MOOSEDocs system underwent periodic updates. 


\subsection{Standardization of Assessment Case Plots}

The plots which compare Bison simulation results against experimental measurement data are an important component of the LWR validation documentation. These plots concisely describe the capability of Bison to capture fuel rod performance behavior. During the FY19 we have developed a standard style and format guide for the assessment case result plots and have updated a majority of these plots within the Bison LWR assessment suite documentation. The uniform appearance of the standardized plots provides better readability and clarity for users. To enforce the uniform plot appearance, we have developed a centralized plotting style-file to standardize all data-visualizations within the documentation. We have also laid the groundwork to automate all validation case plots to update with the most recent Bison simulation results data.

\subsubsection{Matplotlib Style-File}

Previously the Bison LWR validation case documentation contained graphics generated from an array of different software; these static plots were created with ad-hoc programs that were easily accessible. While the information contained within the plots is valuable, the visual aesthetic created a stark discontinuity between respective validation case reports. In addition to the scattered appearance of the documentation, the varying visual aesthetics from the use of different fonts, colors, and legend formats could negatively impact the documentation usability. To increase the readability and uniform appearance of the plots across the different assessment case documentation pages, we designed a centralized plot style-file to enforce a consistency in visualizations across the documentation.

We decided to standardized the plots with python and matplotlib. With these coding libraries, we were able to design a file that all plots within the documentation would query to give them similar appearances. This style file will continue to be updated as needed. At present, it contains the following features:

1. Consistent fonts and font sizes.

2. Distinct and larger legends

3. Color-blind accessible color-palette

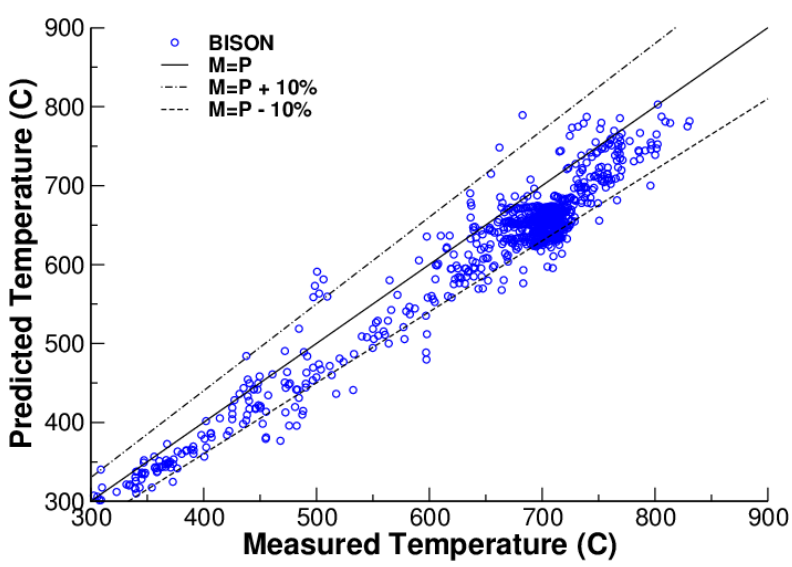

(a) Legend and font sizes before applying the new style-file

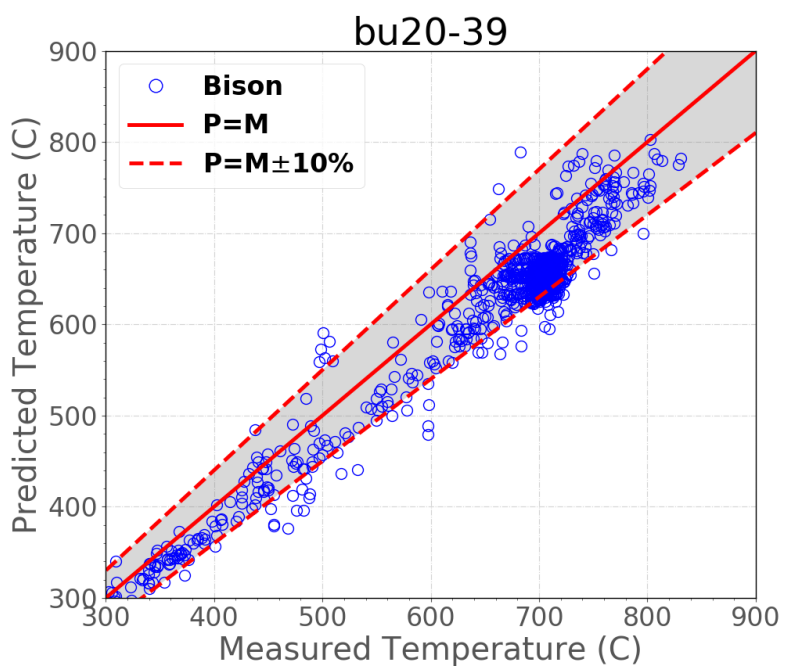

(b) Legend and font sizes after applying the new style-file

Figure 13: Comparison of Bison assessment case plot readability before and after the application of the style-file.

Figure 13 displays some of the effects of the updated style-file. The legend has been simplified and enlarged, colors and shading have been utilized to allow the reader to distinctly see the confidence boundary. This is just a brief example of how the validation case plots have been updated to be more clear to the reader and consistent across Bison's documentation. 
Within the style file we have also opted to utilize a color-blind accessible color palette. Over $8 \%$ of males, and $0.6 \%$ of females are color blind in some shape or form [8]. By using a particular color-palette, Bison's visualizations can reach a broader audience and prevent confusion among those people who cannot distinguish between particular color groups.

We consulted an online color-palette generator, ColorBrewer.org, that generates a color palette accessible to all forms of color-blindness [9]. Our style-file automatically rotates through these colors while generating a graphic with distinct variables. Since many plots only contain one to three variables, the first three colors are starkly contrasted in red, black, and blue. As a plot requires more variables, the colors branch out strategically to avoid clashing colors and colors not visible to those with color-blindness. Figure 14 shows Bison's color palette in the order that the colors are selected, while Figure 15 shows the color palette in action.

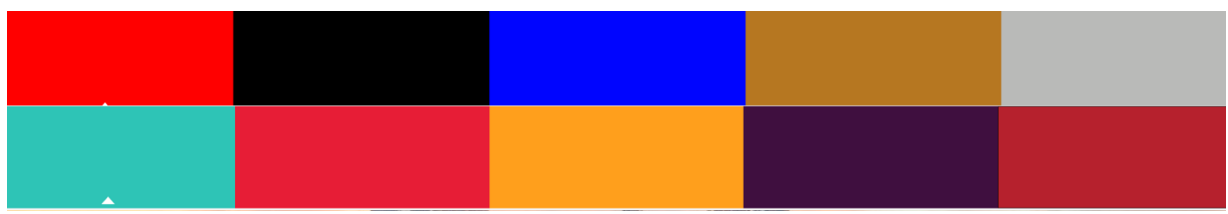

Figure 14: The color-blind accessible color palette implemented within the Bison plot style guide ensures a wider audience can easily understand the LWR assessment suite documentation plots. The style guide uses colors from the top row, left to right, before selecting colors from the bottom row, also in a left-to-right order.

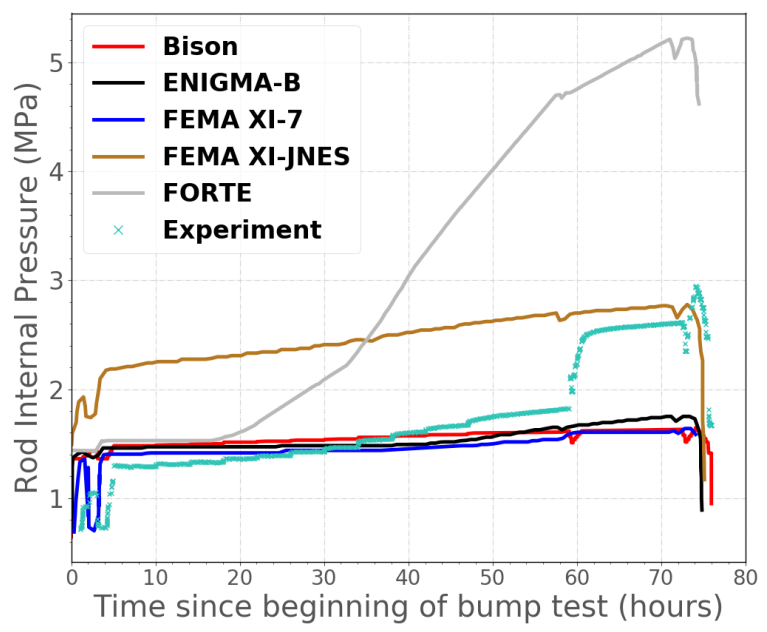

Figure 15: Example of the standard Bison color-palette in use for a graph with many data sources.

\subsection{Modernize Plots in Assessment Cases Documentation}

With the plot standardization guidelines formalized in the style guide, we have begun work on updating all the plots within the assessment case testing suite to reflect the current state of Bison's source code. Many plots in the documentation were generated before new features, additions, and models were utilized. In combination with updating the text to discuss the newly implemented tensor mechanics classes, the plots will be updated to reflect the output of the tensor mechanic models.

There are over 85 assessment case documentation pages within the testing suite, many containing over 12 plots. We note that one assessment case and the corresponding documentation page may be associated with multiple input files. For example, the Ris $\varnothing$ II 3 assessment case includes two assessment case input files (one in solid mechanics and one in tensor mechanics) while the REBEKA LOCA assessment case includes 19 input files (9 mechanics input file pairs and 1 Layered1D input file). At the time of this writing, approximately $70 \%$ of the assessment cases have been updated to contain current visualizations. 


\subsubsection{Initial Automated Plot Generation Development}

Given the ad-hoc nature of previously generated plots, we are currently working on a reproducible, step-by-step documentation of how to generate these plots. We have not yet been able to reproduce some plots because some experimental data was briefly gathered and cleaned on an employee's local computer. The results of our current efforts will include storing all of the plotting data in the Bison repository. An excel workbook, containing information for each plot within the assessment case, will also be added to the Bison repository. These efforts will aid us in our future work of automating our documentation systems.

Currently we are investigating the automating the plot generation for the Bison LWR assessment suite documentation. By utilizing a python script and a nightly post-test specification file, an automation script will fetch the most recent Bison run data and combine the simulation results with experimental data. The automation script will then update the plot and run an image-diff algorithm against the previously gold standard plot. If the image is significantly different from the accepted plot, the Bison development team will be notified to determine if the plot should be updated within the documentation. 


\section{Development of New LWR Validation Cases}

\subsection{IFA-629.4 High Burnup Fuel Case}

The purpose of the Halden IFA-629 test series was the production of data to improve understanding and modeling of high burnup fuel behavior through the concurrent measurement of fuel temperature, fission gas release and rod elongation during both steady operation and controlled power ramps. The fourth loading of IFA-629, considered here, utilized two $\mathrm{UO}_{2}$ rods base-irradiated for five cycles in the French Gravelines-5 PWR to burnups of 54.7 (rod 7) and $54.2(\operatorname{rod} 8) \mathrm{MWd} / \mathrm{kgUO}_{2}$. Although comparison of Bison predictions to fuel temperature and pressure are included here, the main interest in this study was rod elongation. Frictional contact capability was recently added to Bison (prior models assumed frictionless contact between the fuel and cladding) thus this comparison represents a first validation to rod elongation data.

\subsubsection{Experimental Description}

Although the IFA-629 rig can accommodate up to four short length re-instrumented fuel rod segments, only two rods were used in the fourth loading, and both were fitted with fuel centerline thermocouples [10]. Rod 7, in Rig Position 1, was equipped with a clad elongation detector, and Rod 8, in Rig Position 3, contained a pressure transducer. Table 1 provides details on the refabricated characteristics of the fuel rods and reactor operating conditions. Note that Rods 7 and 8 were essentially identical with only a small difference in the base irradiation burnup.

Table 1: Characteristics of the IFA-629.4 fuel rods and operating conditions [10].

\begin{tabular}{lll} 
& Rod 7 & Rod 8 \\
\hline Fuel material & $\mathrm{UO}_{2}$ & $\mathrm{UO}_{2}$ \\
Base irradiation burnup $\left(\mathrm{MWd} / \mathrm{kgUO}_{2}\right)$ & 54.7 & 54.2 \\
Fuel density $(\% \mathrm{TD})$ & 95.32 & 95.32 \\
235U Initial enrichment (wt \%) & 4.487 & 4.487 \\
Fuel grain size $(\mu \mathrm{m})$ & 9.3 & 9.3 \\
Active fuel length $(\mathrm{mm})$ & 441 & 441 \\
Pellet OD $(\mathrm{mm})$ & 8.192 & 8.192 \\
Pellet ID $(\mathrm{mm})$ & 0 & 0 \\
Pellet length $(\mathrm{mm})$ & 13.78 & 13.78 \\
Pellet dish diameter/depth $(\mathrm{mm})$ & $6.00 / 0.32$ & $6.00 / 0.32$ \\
Pellet shoulder width $(\mathrm{mm})$ & 0.56 & 0.56 \\
Cladding material & $\mathrm{Zr} 4$ & $\mathrm{Zr} 4$ \\
Cladding ID (mm) & 8.36 & 8.36 \\
Cladding OD (mm) & 9.50 & 9.50 \\
Initial diametral gap $(\mu \mathrm{m})$ & 170 & 170 \\
Plenum volume $\left.(\mathrm{cm})^{3}\right)$ & $3.65(\mathrm{calc})$ & $3.65(\mathrm{calc})$ \\
Fill gas & $\mathrm{He}$ & $\mathrm{He}$ \\
Fill gas pressure $(\mathrm{MPa})$ & 2.6 & 2.6 \\
Coolant pressure $(\mathrm{MPa})$ & 3.4 & 3.4 \\
\hline
\end{tabular}

Radial and axial power distributions were mapped using an array of six vanadium neutron detectors. Experiments were performed under mild over-power conditions, rather than severe power ramps which could lead to rod failure. As emphasized in [10], these conditions are of more value in the study of fuel performance since the occurrence of fuel failure precludes any subsequent post-irradiation examinations. A series of power ramps, including several scrams, were used to assess steady and transient thermal and PCMI behavior.

\subsubsection{Bison Computational Model}

The geometric parameters specified in Table 1 were used to develop a 2D axisymmetric finite-element mesh that suitably represents the experimental rods, including individual fuel pellets, cladding tube, and plenum volume. For simplicity, both the commercial base irradiation and power ramping in the Halden reactor were simulated using the refabricated 
rod geometry. Figure 16 shows a section of the mesh near the top of the pellet stack, where the dished pellets transition from solid (red) to annular (yellow). The mesh has been magnified 2x in the radial coordinate to improve visualization. The annular pellets are designed to accommodate the thermocouple tube that extends out the top of the rod. Linear quadrilateral finite elements were used for both the fuel and cladding materials. The initial plenum length was adjusted such that the plenum volume at refabrication time was approximately the calculated volume of $3.65 \mathrm{~cm}^{3}$ as specified in Table 1. The adopted mesh parameters are consistent with previous Bison solution verification studies [3] that demonstrate reasonable accuracy of the Bison thermo-mechanics solution.



Figure 16: A section of the finite element computational mesh near the top of the pellet stack where the dished pellets transition from solid (red) to annular (yellow). The mesh has been magnified $2 \mathrm{x}$ in the radial coordinate to improve visualization.

$\mathrm{UO}_{2}$ thermal behavior was computed using the NFIR thermal conductivity model [4] assuming a constant thermal expansion coefficient of 10e-6 (1/K). In order to evaluate the effects of different fuel mechanical models, calculations were performed assuming: 1) elastic fuel without cracking, 2) creeping fuel with isotropic cracking, and 3) creeping fuel with smeared cracking. Isotropic cracking and fission gas release were approximated using the models described in [11] and [12], respectively. The $\mathrm{Zr}-4$ cladding was assumed to have constant thermal conductivity and thermal expansion coefficients of $16 \mathrm{~W} / \mathrm{m}^{2}$ Kand $5 \mathrm{e}-6(1 / \mathrm{K})$, respectively. Cladding thermal and irradiation creep were modeled using the models of Limbäck and Hoppe, respectively, as described in [4].

Thermal contact was modeled assuming both conductance through the gas gap and increased conductance as a function of fuel-cladding contact pressure, as described in [4]. Note that the standard surface roughness values for the fuel and cladding were reduced by a factor of roughly 10 to approximately account for the oxide layer that grows between the fuel and cladding, eventually bonding the materials at high burnup. Mechanical frictional contact between the fuel and cladding was modeled using a hybrid approach recently implemented in Bison. The hybrid method enforces kinematic constraint in the radial direction and penalty constraint in the axial direction, using a procedure to limit the size of a nonlinear iterative solution update. This method deals better with nonlinearities due to reversals in the slip direction at nodes in contact. This approach has clearly improved the robustness of frictional contact in Bison, however, further development and refinement is needed.

The base irradiation power history was developed previously in conjunction with a PCMI benchmark exercise, described in part in [13]. The average power history was approximated by constant power operation at 20, 25, 24, 21, and $17 \mathrm{~kW} / \mathrm{m}$ for cycles of length $6088,7327,6237,6383$ and 6176 hours. Note that at the end of the base irradiation, 
the average burnup computed by Bison for Rod 7 was $52.7 \mathrm{MWd} / \mathrm{kgUO}_{2}$, within a few percent of the desired value of $54.7 \mathrm{MWd} / \mathrm{kgUO}_{2}$ given in Table 2. Refabrication at the end of base irradiation was accomplished by refreshing the rod fill gas to pure $\mathrm{He}$ at ambient temperature and a pressure of 2.6 MPa.

In conjunction with the PCMI benchmark mentioned above, the power history for Rod 7 was condensed from detailed Halden data by JRC-Karlsruhe using the Fuel Rod Analysis Toolbox software [14]. Although four separate histories were provided based on axial location (average and lower, middle and upper third of the rod) the curves differed by only $1 \mathrm{~kW} / \mathrm{m}$, thus the average value was used and a flat axial profile was assumed. This history will be shown below as comparative results are presented.

As part of the data condensation effort, a bulk coolant temperature history was also provided by ITU and assumed applicable over the entire length of the short rodlet. As suggested for the PCMI benchmark, the rod surface temperature at any given elevation was then computed as the bulk coolant temperature plus the Jens-Lottes film temperature drop [15] at that elevation.

Bison simulations were run for a variety of conditions to enable parametric analysis. As mentioned above, calculations were performed assuming either elastic fuel without cracking, or creeping fuel with either isotropic cracking or orthotropic smeared cracking. The isotropic cracking model is empirical and very simplistic, assuming an isotropic reduction in elastic modulus as a function of the power level. Additionally, for each fuel mechanical behavior assumption, calculations were run with a variety of pellet to cladding friction factors, ranging from 1000 to 0.1 .

\subsubsection{Results and Comparisons}

Figure 17 shows a comparison of the computed and measured fuel centerline temperature and rod pressure during the Halden irradiation. Also shown are the measured power histories for Rods 7 and 8, compared to the condensed Rod 7 history used as input to Bison; the measured and condensed power histories for Rod 7 overlay, as expected. Note that while only Rod 7 was simulated with Bison, comparisons are made to temperature measurements for both rods, and to the measured rod pressure ( $\operatorname{Rod} 8)$. These comparisons are reasonable given that the geometry, burnup and power histories for the two rods are very similar.
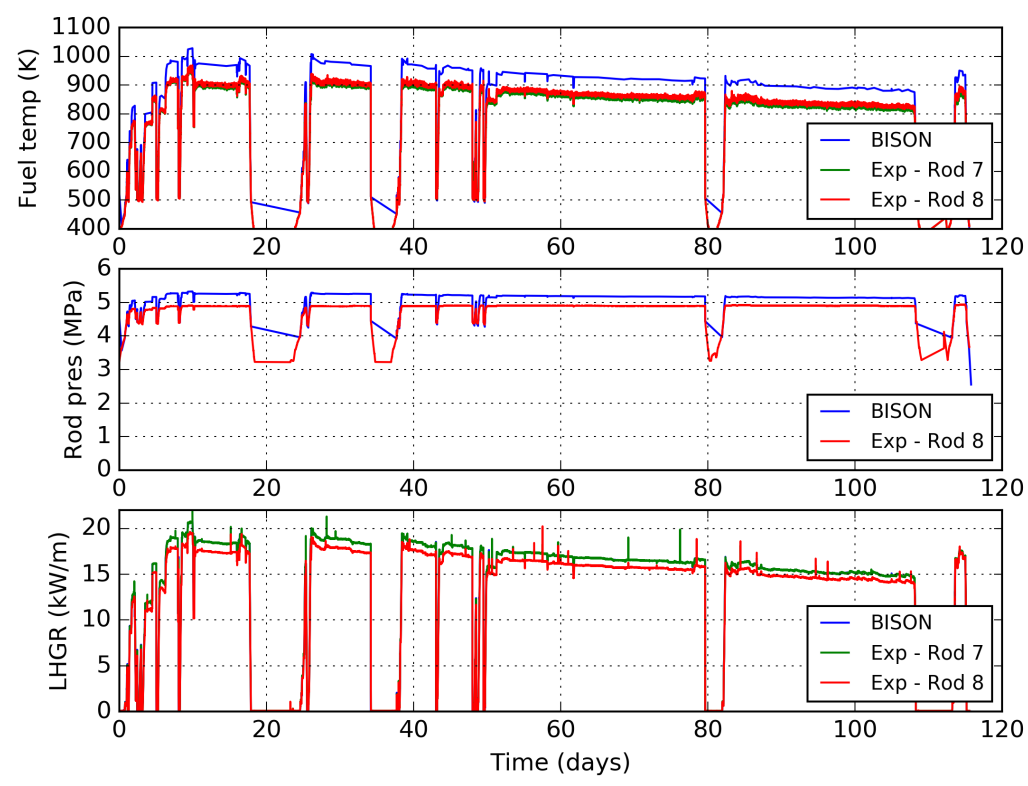

Figure 17: Comparison of the computed and measured fuel centerline temperature and rod pressure during the Halden irradiation. Also shown are the measured power histories for Rods 7 and 8, compared to the condensed Rod 7 history; the measured and condensed power histories overlay. 
The predicted temperature is systematically roughly $70 \mathrm{~K}$ above the measured values, which is not unreasonable given the uncertainties involved in both the calculation and measurements. It is interesting to note that although the power history for Rod 7 is systematically larger than that of $\operatorname{Rod} 8$, the measured temperature of Rod 7 is systematically lower than Rod 8 even though the rods are essentially identical, possibly providing insight into measurement uncertainty. We note that [10] suggests that the presence of the molybdenum thermocouple tube results in a neutron flux depression and localized reduction in power (approximately $2 \%$ ) in the region where fuel temperature comparisons are made. For consistency with prior comparisons to Halden fuel centerline temperature measurements, this correction was not applied here, but would result in an improved comparison between prediction and measurement. Finally, refining the calculation by including an axial power profile will provide better temperature agreement since the measured power in the top third of the rod was approximately $3 \%$ lower than the average value used in the simulation.

The comparison of measured and predicted rod pressure in Figure 17 is very reasonable, again considering the associated uncertainties. In [10] the measured pressure history was used to estimate fission gas release during the Halden irradiation, which was determined to be less than $1 \%$. The predicted fission gas release during this period was essentially zero $(0.0005 \%)$.

The Rod 7 power history for the first 10 days of Halden irradiation, displayed in Figure 18, shows that the rod average power is increased in a series of ramps and holds (identified as Phases) each occurring over approximately 2-3 days. Rod elongation comparisons reported here focus on the Phase 2 ramp identified in the figure.

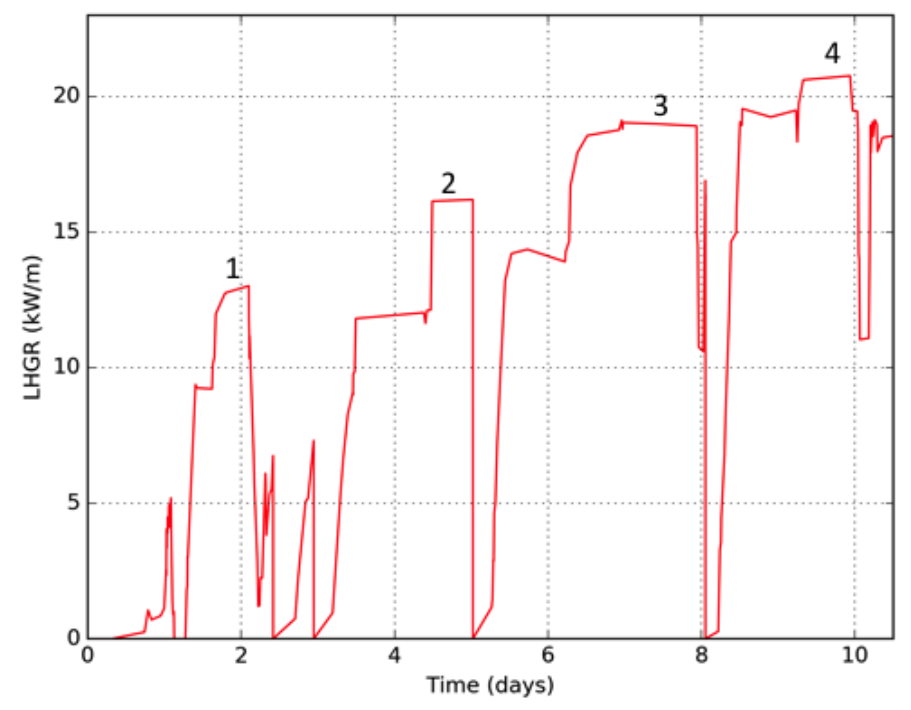

Figure 18: Rod 7 power history for the first 10 days of Halden irradiation.

The predicted and measured rod elongation, as a function of rod average power during the Phase 2 power ramp, are shown in Figure 19. Results are shown for purely elastic fuel and creeping fuel assuming either isotropic or smeared cracking. Note that an estimate of the fuel thermal expansion slope, taken from [10], is included for reference and was computed based on approximation of the fuel temperature change at the inner radius of the lands between adjacent fuel pellets. Results are shown assuming a fuel-clad friction factor of 0.3 ; however, additional simulations with friction factors varying between 0.1 and 1000 did not indicate a strong sensitivity to friction over that range. 


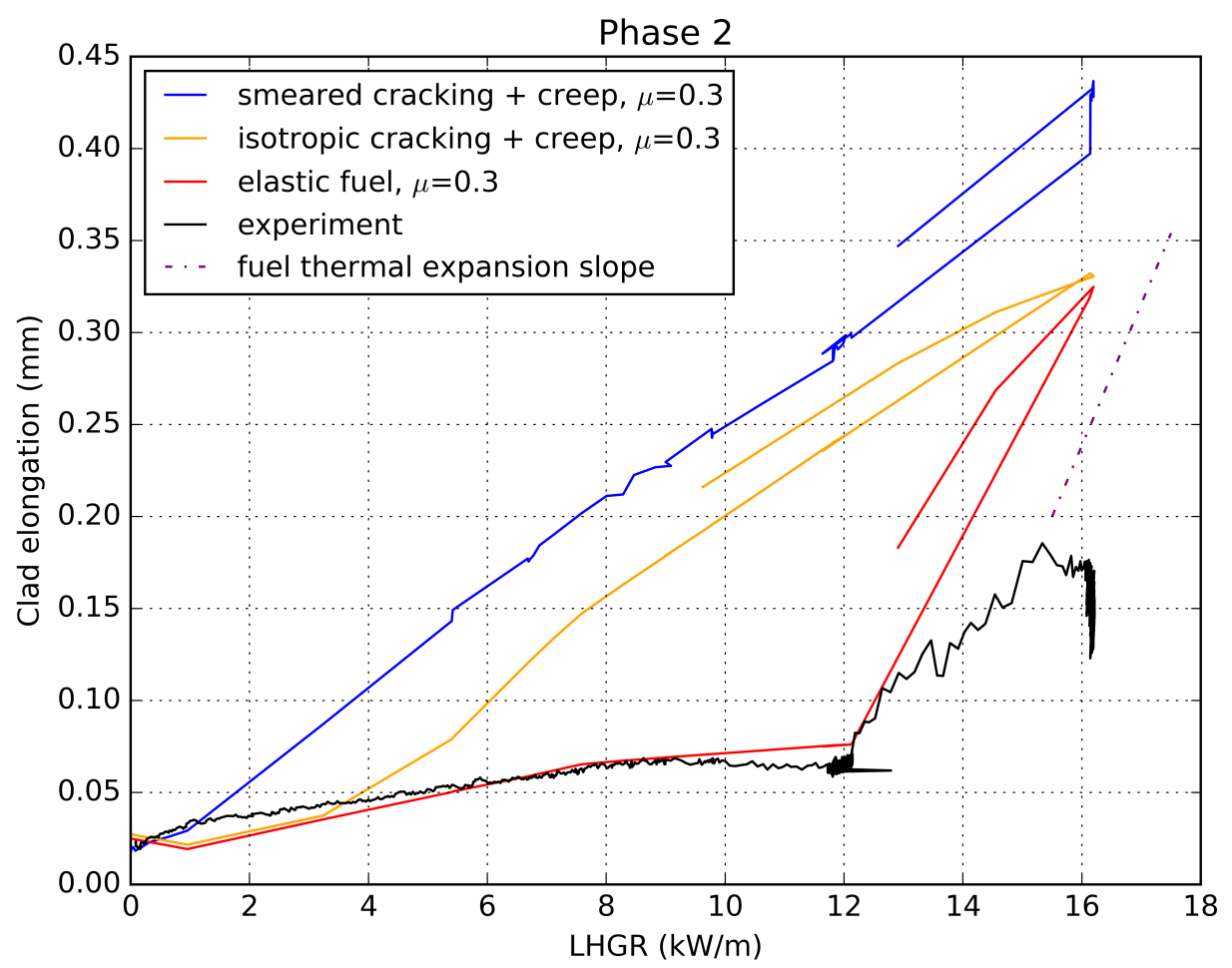

Figure 19: The predicted and measured rod elongation, as a function of rod average power, during the Phase 2 power ramp. Predictions are shown assuming elastic fuel and creeping fuel with either isotropic or smeared cracking material models.

Both the experimental data and elastic fuel simulations suggest three different elongation slopes, which are likely correlated to different contact conditions between the fuel and cladding (namely non-contact, intermediate-contact, and hard-contact). During non-contact, the rod elongation simply follows the thermal expansion of the cladding. During intermediate-contact there appears to still be slip between the fuel and cladding, however, the cladding diameter increases slightly as driven by radial fuel thermal expansion, resulting in a flattening of the axial elongation slope. Following the onset of hard-contact at approximately $12 \mathrm{~kW} / \mathrm{m}$, the cladding is locked (or partially locked) to the fuel and thus the predicted rod elongation reflects the fuel thermal expansion. This explanation generally follows that given in [10], where the IFA-629.4 experimental results were analyzed in detail.

For the Phase 2 ramp with elastic fuel, the computed and measured elongations correlate well during both the non- and intermediate-contact phases, with the onset of hard-contact well-predicted. There are, however, significant discrepancies in both the elongation slope and magnitude during the hard-contact period. Bison tends to follow the fuel thermal expansion slope (dashed purple line), as expected, while the measurement indicates a lesser slope. The expected change in elongation that occurs during the second power increase (between points A and B in Figure 18) can be estimated using the Bison-predicted change in fuel temperature at the inner land location. That temperature difference is approximately $40 \mathrm{~K}$ which, when multiplied by the active fuel length $(441 \mathrm{~mm})$ and thermal expansion coefficient $(10 \mathrm{e}-61 / \mathrm{K})$, results in an elongation of $0.27 \mathrm{~mm}$, very similar to the predicted values in Figure 19. Differences in the measured and predicted elongation slope and magnitude indicate that other physics, not included in the elastic fuel calculation, must be in play. The oscillations in the measured elongation, which seem real when compared to measurement noise prior to hard-contact, suggest there could be periodic slip occurring between the fuel and cladding. Another possibility is axial fuel compaction, possibly by the closure of axial cracks, resulting from the force imposed by the cladding. 
It is clear in Figure 19 that the simulations which included fuel creep with either isotropic or smeared cracking do not predict the measured rod elongation behavior well, with the onset of hard-contact occurring much too early in the power ramp. It is interesting to note, however, that once contact is established, the rate at which the elongation increases with power (the slope of the line) is quite similar to that measured. The results suggest that elastic fuel behavior is appropriate to establish the correct fuel-cladding gap behavior leading up to hard contact, but then less stiff fuel, as given by the isotropic and smeared cracking models, is appropriate during hard contact. This possibility will be further investigated, first by comparing predicted and measured results for additional power ramps for this validation case.

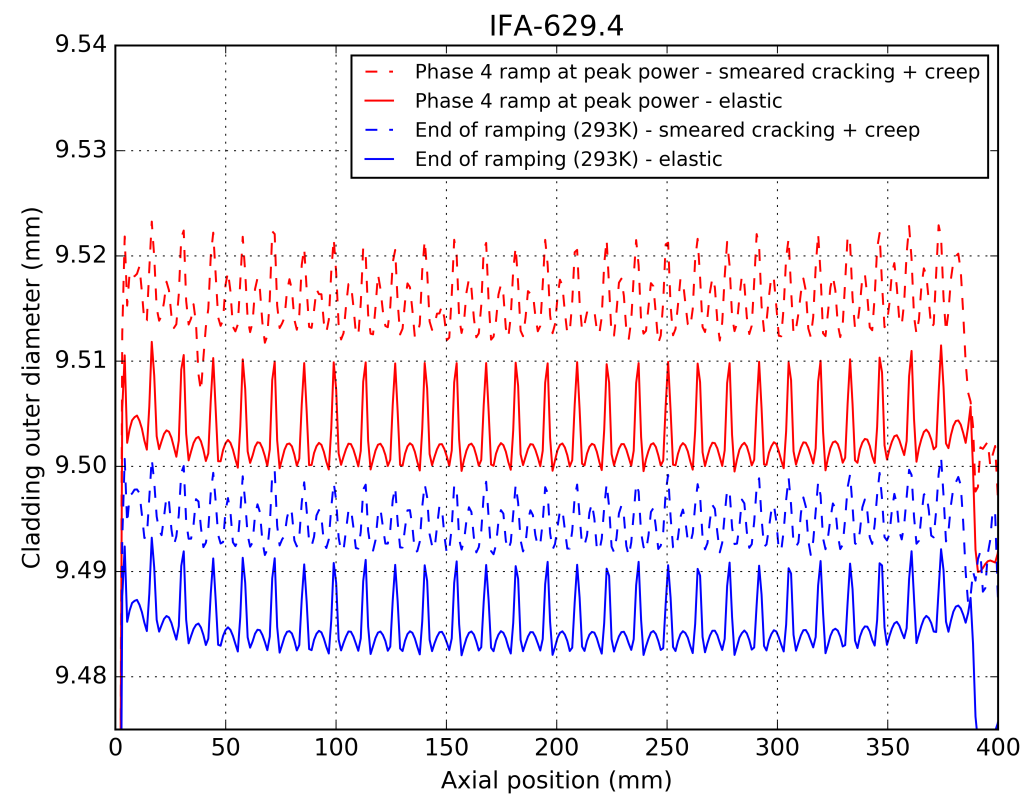

Figure 20: Computed cladding outer diameter over the active fuel length, at different times in the calculation.

The computed cladding outer diameter over the active fuel length, at different stages in the calculation, is shown in Figure 20. Ridges in the cladding at each pellet-pellet interface result from the hourglass shape of the fuel pellets. Note that the annular pellets at the top of the stack show significantly less ridging. Although PIE cladding diameter measurements are not available for this experiment, computed end-of-life ridge heights of 7-10 $\mu \mathrm{m}$ are reasonable based on similar experiments. Finally, note that including fuel creep results in an additional ridge at the waist of each fuel pellet, a phenomenon that has been observed in earlier studies. 


\section{Expansion of User Support Documentation}

In the final focus area of this milestone our efforts centered on expanding and improving the Bison documentation geared towards user support. These efforts differ from those assessment case documentation updates discussed in Section 3 in that these documentation expansions focus on general Bison use. As in the assessment suite documentation, these general use documentation pages require regular revisions to ensure continued compatibility with the Bison source code. In this section of the report we describe the revisions to the Bison getting started guide, and the development of an input file migration guide.

\subsection{Revised Bison Getting Started Page}

The revision of the getting started portion of the Bison documentation was conducted in response to user feedback about difficulties with installing and building the Bison code. Here we refer to installation as the process of downloading the code and setting up the correct environment on the machine while building is the process of creating the Bison executable. The previous installation and build guide relied on a user to switch between several different pages and to identify which particular sections of the individual pages were relevant to the user's own situation. This page navigation assumed an intermediate familiarity with Unix based operating systems. For new Bison users who were transitioning from a Windows operating system, these navigation requirements posed a significant challenge. These difficulties may have discouraged users in the past.

The changes we have made in FY19 to the Bison getting started documentation focus on providing a single unified set of instructions. The introduction page for the getting started section, shown in Figure 21, immediately clarifies the potential different user situations. Once a user selects one of these options they are transferred to a page with the complete set of installation and building instructions.

\section{Choose the option below that best fits your environment and situation:}
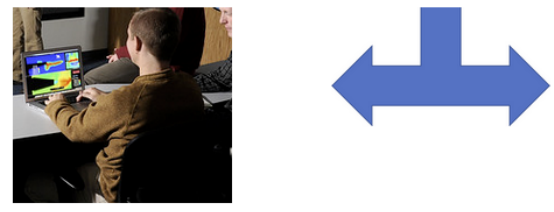

Building on a local machine

New user first time Bison download

New computer Bison download

Updating your Bison



Building on the INL HPC

New user first time Bison download

New HPC directory Bison download

Updating your Bison

Figure 21: The improved Getting Started page of the Bison documentation reduces confusion for users by clarifying separate installation and build paths for different use situations. Each link in the documentation excerpt shown here leads to a continuous and inclusive set of installation and building instructions.

Intermediate and returning experienced users also benefit from the getting started section redesign. The lower links on the introduction page, Figure 21, are geared towards these users who may require assistance with updating Bison or moving to a new machine. The new links help these continuing Bison users quickly identify and navigate to information relevant to their situation. For both new and continuing Bison users, these documentation changes make significant progress towards reducing the difficulties with installing and building Bison. 


\subsection{Tensor Mechanics Migration Guide Development}

To aid in the assessment case input file migration to tensor mechanics, we have developed a mechanics migration guide. Unlike the getting started guide, the migration guide is geared towards experienced Bison users, including internal INL users. The mechanics migration guide is intended to help these users in migrating existing Bison LWR input files to the new tensor mechanics system.

The mechanics migration guide outlines the procedure we have developed as shown in Figure 22. Many components of this procedure, such as the StandardLWRFuelRodOutputs action and the standard comparison plot script, are described in other sections of this report.

\section{Add the StandardLWRFuelRodOutputs Action}

Before starting the mechanics migration process, ensure that your SolidMechanics input file includes a standard set of output quantities, with standardized names, for easier comparison of simulation results during the migration process. In this step the StandardLWRFuelRodOutputs Action is introduced and the process of adding the action into an existing SolidMechanics input file is discussed.

Review detailed information on this step here.

\section{Duplicate the SolidMechanics Input File}

Begin by copying over the SolidMechanics input file modified in Step 1 above to create the basis of the new TensorMechanics input file. In this step the naming conventions and the additional files required to run the new TensorMechanics input files within the INL idaholab repository testing harness are also discussed.

Review detailed information on this step here.

\section{Convert the Mechanics Input File Blocks}

Use the series of correspondence tables and examples given in this step to replace the SolidMechanics specific input file blocks with the correct TensorMechanics blocks. This step is the longest and most difficult in the process of migrating an input file and may take several days. Ensure that both the SolidMechanics and the TensorMechanics input files both run to completion before proceeding to the next step.

Review detailed information on this step here.

\section{Compare Results with Standardized Plots}

A successful migration from the solid mechanics module to the tensor mechanics module system is completed when the simulation results are replicated. One the best ways to compare the results of the tensor mechanics simulation to the solid mechanic simulation is with a set of plots.

Review detailed information on this step here.

\section{Investigate Differences in the Simulation Results}

Review detailed information on this step here.

Figure 22: An excerpt from the Bison online documentation, the overview of the mechanics migration guide lists the five main steps to take when migrating an existing Bison LWR input file from solid mechanics to tensor mechanics. 
The plug-n-play nature of the tensor mechanics module complicates the input file migration process. Because extra care is required in the migration of the [Materials] input file blocks, we have included detailed examples of corresponding solid mechanics and tensor mechanics material blocks in the guide, see Figure 23. Although the tensor mechanics system does lengthen the input file, we reiterate that the tensor mechanics system gives users direct control over which material models are used in the Bison simulation.

\title{
Complete Migration Comparison
}

\begin{abstract}
Review the complete migration of the solid mechanics Zircaloy mechanics material conversion with the example shown below before proceeding to the next step. Remember that the tensor mechanics input file blocks may be different from the example shown based on the parameter settings in the solid mechanics input file block.
\end{abstract}
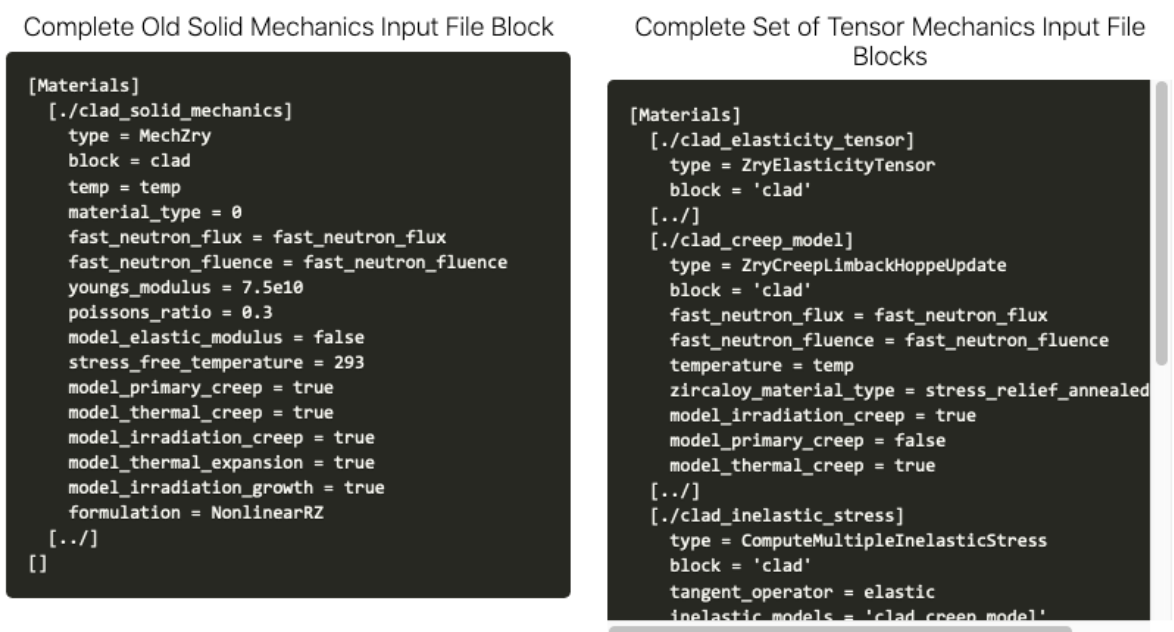

Figure 23: This excerpt from the tensor mechanics migration guide is an example of the side-by-side comparison of the equivalent solid mechanics and tensor mechanics material blocks.

The mechanics migration guide also includes a description of how to compare the results of the migration tensor mechanics and original solid mechanics simulations. For the situations in which the two mechanics simulations do not match, the guide offers suggestions about potential migration pitfalls, such as different defaults or strain formulations, including those we discussed previously in Section 2.3.

While the mechanics migration guide does not cover every solid mechanics material in the Bison source code, we have focused on those materials that are generally used in a Bison LWR simulation. Users are encouraged to contact the Bison developers for those specialized cases not covered in the migration guide. Additionally the many mechanics pairs of the input files included in the Bison LWR assessment suite are supplemental examples of the input file migration process. 


\section{Summary and Future Work}

In FY19 we have made several improvements to the Bison validation base, separating our efforts into updates to the existing LWR assessment suite and new additions to Bison. We have further categorized both of these topics into two focus areas: one focus area centers on the simulation capabilities of Bison and the associated input files while the second focus area is concerned with documentation improvements.

A majority of our efforts for FY19 were focused on improvements to the existing Bison LWR validation suite. These improvements included a significant effort to migration the Bison LWR assessment suite to the more reliable and robust tensor mechanics module system. Although code reliability issues in the solid mechanics module have hampered this migration process, we have successfully migrated $64 \%$ of the LWR assessment input files to use the tensor mechanics system. Additionally we have completed work to standardize the input files and to investigate the impact of additional physics models for the fuel in PCMI assessment cases. Our initial investigation with these new fuel physics models indicates that these models could improve Bison simulation predictions of radial clad displacements in PCMI validation cases. As an accompaniment to these Bison validation input files, we have also completed revisions of the validation documentation with an emphasis on standardizing the simulation result plots. These uniform plots will provide better readability and clarity for users reviewing the current Bison LWR assessment suite.

Our new addition to the Bison validation suite is the development of the IFA-629.4 High Burnup Fuel case. This assessment case is the first case in the Bison suite to use frictional contact, with a primary focus on modeling the rod elongation measured at Halden. The pressure and fuel centerline temperature predictions from Bison align with the experimental measurements, and ongoing investigations are being conducted to better understand the rod elongation mechanisms. We have also expanded the user support focused documentation in Bison. Our documentation expansion efforts have included a revision of the Bison installation instructions and the creation of a tensor mechanics migration guide. These documentation additions will reduce some of the barriers to Bison use for both new and existing users.

Future developments and improvements for the Bison validation base will continue to focus on ensuring the validation input files represent the current capabilities of Bison and on assisting Bison users with harnessing these capabilities. To achieve these goals we have identified the following tasks for future work:

- Complete the process of migrating all Bison validation case input files to the more reliable and robust tensor mechanics system, including user support for external users migrating input files,

- Automate the generation of plots to compare Bison validation simulation results with experimental measurements on a more frequent basis,

- Develop a simplified and condensed Bison input file interface for typical fuel performance simulations, with a focus on reducing barriers to use for potential industry partners who are exploring the idea of using Bison.

All of these future development goals will build on the work completed under this milestone to update, expand, and improve the Bison validation suite and documentation capabilities.

\section{Acknowledgments}

This work was sponsored by the U.S. Department of Energy, Office of Nuclear Energy, under the Consortium for Advanced Simulation of Light Water Reactors (CASL) and Nuclear Energy Advanced Modeling and Simulation (NEAMS) programs.

This manuscript has been authored by Battelle Energy Alliance, LLC under Contract No. DE-AC07-05ID14517 with the U.S. Department of Energy. 


\section{References}

[1] B. W. Spencer, R. L. Williamson, A. D. Lindsay, F. Kong, R. J. Gardner, J. D. Hales, A. Casagranda, D. Schwen, H. Chen, N. Prakash, C. Matthews, and C. Unal. Bison improvements for robustness and speed. Technical Report CASL-U-2018-1625-000, INL/EXT-18-45704, Idaho National Laboratory, June 2018.

[2] S.A. Pitts, H. Chen, B.W. Spencer, S.R. Novascone, J.D. Hales, and R.L. Williamson. Bison documentation expansion for tensor mechanics and layered1d capabilities. Technical Report CASL-U-2018-1644-000, INL/EXT18-45780, Idaho National Laboratory, June 2018.

[3] RL Williamson, KA Gamble, DM Perez, SR Novascone, G Pastore, RJ Gardner, JD Hales, W Liu, and A Mai. Validating the Bison fuel performance code to integral LWR experiments. Nuclear Engineering and Design, 301:232-244, 2016.

[4] Bison manual. https://mooseframework.org/bison/. Technical Report, Release 1.5 INL-MIS-18-50577-Rev001.

[5] Seved Djurle. Final Report of the Super-Ramp Project. Technical Report STSR-32, Studsvik Energiteknik, AB, 1984.

[6] A. Casagranda, L.K. Aagensen, S.R. Novascone, B.W. Jiang, W. Spencer, and D.J. McDowell. Summary of Bison development and validation activities - NEAMS FY19 report. Unpublished, September 2019.

[7] Andrew Slaughter, Cody Permann, Derek Gaston, and Richard Martineau. NEAMS-IPL MOOSE Midyear Framework Activities. Technical report, Idaho National Laboratory, Idaho Falls, ID (United States), 2018.

[8] Karl R Gegenfurtner and Lindsay T Sharpe. Color vision. Cambridge University Press Cambridge, 1999.

[9] Mark Harrower and Cynthia A Brewer. Colorbrewer. org: an online tool for selecting colour schemes for maps. The Cartographic Journal, 40(1):27-37, 2003.

[10] H. Koike, N. Murakami, E. Kolstad, and T. Tverberg. Ramp tests with two high burnup UO2 fuel rods in IFA629.4. Technical Report HWR-769, Halden Reactor Project, 2004.

[11] V. Z. Jankus and R. W. Weeks. LIFE-II - A computer analysis of fast-reactor fuel-element behaviour as a function of reactor operating history. Nuclear Engineering and Design, 18:41-49, 1972.

[12] G. Pastore, L. Luzzi, V. Di Marcello, and P. Van Uffelen. Physics-based modelling of fission gas swelling and release in $\mathrm{UO}_{2}$ applied to integral fuel rod analysis. Nuclear Engineering and Design, 256:75-86, 2013.

[13] G. Rossiter et.al. OECD/NEA benchmark on pellet-clad mechanical interaction modelling with fuel performance codes. In OECD/NEA Workshop on Pellet-Cladding Interaction (PCI) in Water-Cooled Reactors, Lucca, Italy, 22-24 June 2016.

[14] K. Lassmann, A. Schubert, J. van de Laar, and P. Van Uffelen. The 'Fuel Rod Analysis ToolBox': A general program for preparing the input of a fuel rod performance code. Annals of Nuclear Energy, 81:332-335, 2015.

[15] W. H. Jens and P. A. Lottes. Analysis of heat transfer, burnout, pressure drop and density data for high pressure water. Technical Report ANL-627, Argonne National Laboratory, 1951. 